\title{
Behavioural and Psychological Symptoms of Dementia: Correlates and Impact on Caregiver Distress
}

\author{
Adreesh Mukherjee $^{a} \quad$ Atanu Biswas $^{a} \quad$ Arijit Roy $^{b} \quad$ Samar Biswas $^{a}$ \\ Goutam Gangopadhyay ${ }^{a}$ Shyamal Kumar Das ${ }^{a}$ \\ ${ }^{a}$ Department of Neurology, Bangur Institute of Neurosciences and Institute of Postgraduate \\ Medical Education and Research (IPGME\&R), Kolkata, India; ${ }^{b}$ Department of Neurology, \\ Bankura Sammilani Medical College, Bankura, India
}

\author{
Keywords \\ Behavioural and psychological symptoms of dementia - Caregiver distress · Clinical \\ Dementia Rating scale $\cdot$ Neuropsychiatric Inventory
}

\begin{abstract}
Aims: To evaluate the behavioural and psychological symptoms of dementia (BPSD), to determine their correlation with types and stages of dementia and patient demographics, and to assess the impact on caregiver distress. Methods: This cross-sectional study recruited consecutive dementia patients and caregivers who attended our cognitive clinic. Standard criteria were used to classify types of dementia. BPSD were assessed with the Neuropsychiatric Inventory, and its distress scale was used for caregiver distress. Results: Of a total 107 patients, nearly all (99.1\%) had at least one BPSD; $71 \%$ had $\geq 4$ symptoms. Most frequent were apathy and agitation, followed by irritability, sleep and appetite disorders, and mood disorders; disinhibition and euphoria were least frequent. BPSD were less prominent with increasing age; males showed more agitation. Apathy and eating disorders were more prevalent in the rural community. BPSD were highest in frontotemporal dementia (FTD), followed by dementia with Lewy bodies (DLB), and least in vascular dementia. Hallucinations were more common in DLB, aberrant motor behaviour in FTD. All domains of BPSD, except for anxiety and euphoria, were more prominent with increasing severity of dementia. Increasing BPSD (except for euphoria) caused higher caregiver distress. Conclusion: BPSD are universally present, bear correlates with dementia type and severity, and cause significant caregiver distress.
\end{abstract}

(C) 2017 The Author(s)

Published by S. Karger AG, Basel

This work was conducted at the Department of Neurology, Bangur Institute of Neurosciences and Institute of Postgraduate Medical Education and Research (IPGME\&R), Kolkata, India. 


\section{Introduction}

Dementia refers to a significant cognitive decline from a previous level of performance which interferes with independence in everyday activities. However, the "non-cognitive" neuropsychiatric symptoms, or the behavioural and psychological symptoms of dementia (BPSD), are equally important disease manifestations. They are present in a majority of dementia patients and prevail in all types and stages of dementia, although their proportions vary $[1,2]$. The BPSD have several domains which show differences among the various types of dementia, with studies showing predominance of hallucinations in dementia with Lewy bodies (DLB), of depression and apathy in vascular dementia (VaD), of apathy, disinhibition, elation, appetite or eating changes in frontotemporal dementia (FTD), and of apathy, agitation, depression, anxiety, irritability, and sleep disorders for Alzheimer disease (AD) [1, 3-5]. These lead to increased institutionalisation [6] and cause significant caregiver distress [7].

There are studies regarding correlates of BPSD, including disease characteristics and demographic factors, but the results are not always congruent [8] and published data on the prevalence rates of BPSD vary [5]. These contradictions may be due to differing study parameters and definitions or due to variations inherent to the different study populations. With regard to India, there are very few studies on BPSD from this ethnically diverse country [9-12]. Here, we determined BPSD in a hospital-based study population from the eastern region of India, and showed its correlation with various factors, including types of dementia, patient demographics, and other disease characteristics. We also evaluate the effect of BPSD on caregiver distress.

\section{Methods}

\section{Patients}

Consecutive dementia patients, diagnosed as per the Diagnostic and Statistical Manual of Mental Disorders, 4th edition (DSM-IV) criteria [13], attending the cognitive clinic of the institute was included in this cross-sectional study along with their caregivers. The caregiver was considered to be the person who provided regular care/management to the patient and who spent most of the time with the person with dementia. For illiterate patients/caregivers we read out the items (as applicable). The demographic profiles of the patients were noted followed by thorough history and examination. Detailed assessment of cognitive function was performed using standard assessment tools. This was based on the Kolkata Cognitive Battery, the Revised Version of Addenbrooke's Cognitive Examination (ACE-R), and the Frontal Assessment Battery. Additionally, we also tested domains of cognitive functions using standard tests. Every patient was assessed by a single investigator (A.M.), and a consensus diagnosis was made after discussion with other investigators (A.B. and S.K.D.). Relevant haematological, biochemical, and serological investigations were done along with neuroimaging (magnetic resonance imaging of the brain using the Siemens 3 Tesla MAGNETOM $^{\circledR}$ Verio scanner).

Standard criteria were used to classify the dementias: the National Institute of Neurological and Communicative Disorders and Stroke (NINCDS) and the Alzheimer's Disease and Related Disorders Association (ADRDA) (NINCDS-ADRDA) criteria for Alzheimer Disease [14], the International Consensus Criteria for Behavioural Variant FTD [15], the Criteria for the Classification of Primary Progressive Aphasia and Its Variants [16], the Consensus Diagnostic Criteria for Dementia with Lewy Bodies [17], and the National Institute of Neurological Disorders and Stroke (NINDS) Association Internationale pour la Recherche et l'Enseignement en Neurosciences (AIREN) (NINDS AIREN) criteria for Vascular Dementia [18]; mixed 
dementia was considered as a combination of AD with concomitant VaD. Patients with pseudodementia and mild cognitive impairment were excluded from the study. Informed consent for participating in the study was obtained from all participants as well as their caregivers. The study was approved by the institutional ethics committee.

\section{Study Tools}

The staging of dementia severity was performed by the Clinical Dementia Rating (CDR) scale $[19,20]$. There are six domains assessed: memory, orientation, judgment and problem solving, community affairs, home and hobbies, and personal care. Two sets of scores were calculated: Global CDR (CDR-G) scores and CDR sum of boxes (CDR-SOB) scores. CDR-G scores give the following stages of dementia (CDR 0 indicates no cognitive impairment): CDR 0.5, very mild dementia; CDR 1, mild dementia; CDR 2, moderate dementia; CDR 3, severe dementia.

The Instrumental Activities of Daily Living (IADL) were assessed by the IADL in the elderly (IADL-E) scale, and we used the Cognitive Disability Index (IADL CDI) [21]. There are 11 IADL items, and the IADL CDI is derived as the sum of the disability ratings due to cognitive impairment (i.e., cognitive disability score) divided by the product of the maximum possible "disability" on an item (i.e., 2) and the number of items reported applicable.

Assessment of the BPSD was performed with the Neuropsychiatric Inventory (NPI), which was originally developed by Cummings et al. to provide a means of assessing neuropsychiatric symptoms of patients with AD and other neurodegenerative disorders [22, 23]. Ten behavioural (delusions, hallucinations, agitation/aggression, depression/dysphoria, anxiety, elation/euphoria, apathy/indifference, disinhibition, irritability/lability, and aberrant motor behaviour) and two neurovegetative areas (sleep and nighttime behaviour disorders, and appetite and eating disorders) are included in the NPI. We used the 12-item score.

The distress scale of this instrument (NPI-D) [24] was used to assess the caregiver distress, for which the total distress score was generated by adding together the scores of the individual NPI distress questions, and we used the 12-item score. We had previous experience of using the NPI in our population [25].

\section{Statistical Analysis}

Categorical variables are expressed as number and percentage and compared across the groups using the Pearson $\chi^{2}$ test for independence of attributes. Continuous variables are expressed as mean \pm standard deviation and were compared across groups using the MannWhitney U test or the Kruskal-Wallis test as applicable. Associations between continuous variables were obtained by the Spearman rank correlation coefficient. Multivariate analysis was performed using a multiple linear regression model to capture the impact of individual BPSD domains on caregiver distress (NPI-D). The statistical software SPSS version 20 was used for the analysis. Any $p$ value $\leq 0.05$ was taken to be statistically significant.

\section{Results}

\section{Demographic and Clinical Profile}

A total of 107 consecutive dementia patients were included in the study. The different types of dementia were AD 61.7\%, FTD (all behavioural variant FTD) 17.8\%, VaD 10.3\%, mixed dementia 6.5\%, and DLB 3.7\%. Mean age was 66.6 \pm 9.36 years (range 36-90 years). FTD patients were younger $(p=0.005)$; rural patients were younger than urban residents $(p=0.006)$. While $65.4 \%$ of the cohort were male, $71 \%$ were urban residents. The level of 


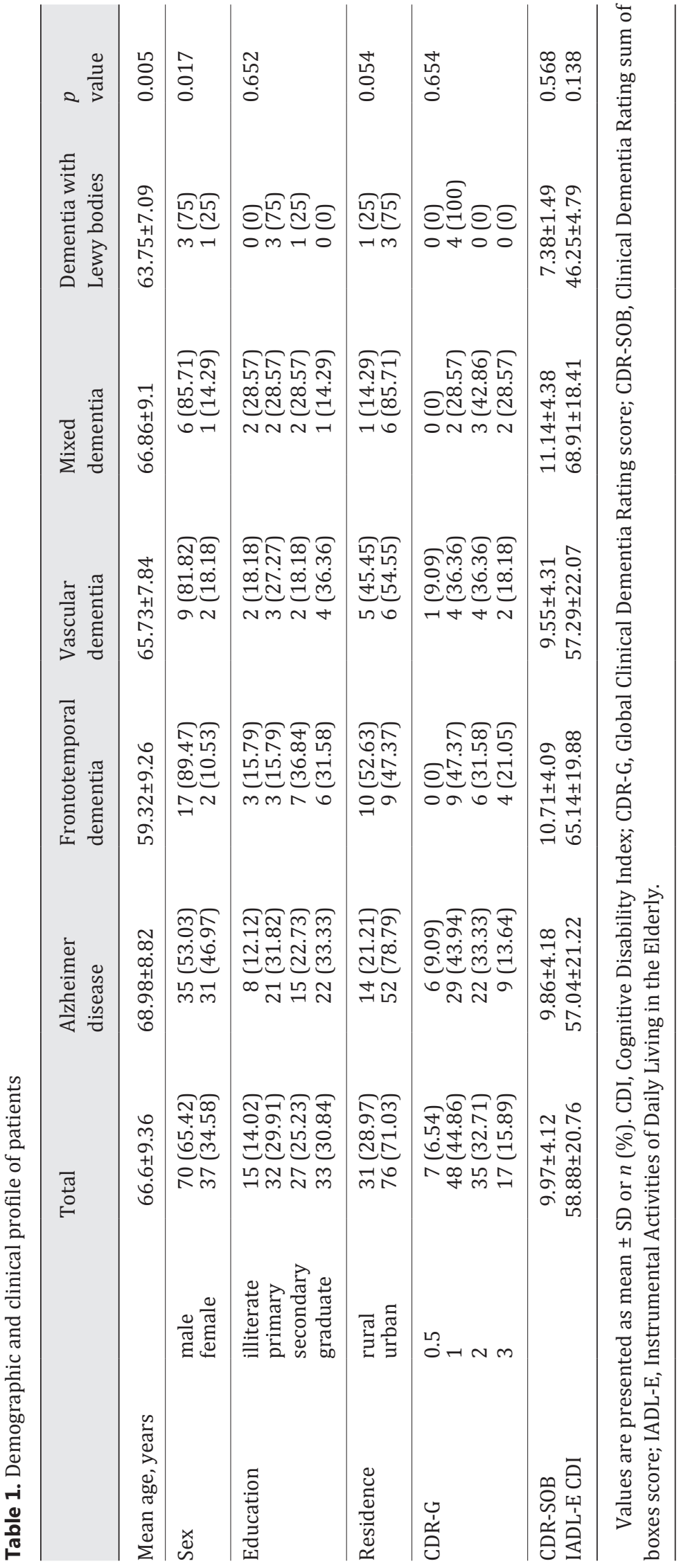


education $(p=0.652)$ and community of residence $(p=0.054)$ were similar among the different types of dementia; however, there were more female patients in the AD group ( $p=$ 0.017) (Table 1).

The mean CDR-SOB score was $9.97 \pm 4.12$. According to CDR-G stages, $6.5 \%$ had very mild dementia (CDR-G score 0.5 ), 77.6\% had mild to moderate dementia (CDR-G scores 1 and 2), and $15.9 \%$ had severe dementia (CDR-G score 3). The mean IADL CDI score was $58.88 \pm$ 20.76. The severity of dementia ( $p=0.568$ for CDR-SOB, $p=0.654$ for CDR-G) and impairment of IADL ( $p=0.138$ ) were similar among the different types of dementia.

\section{Neuropsychiatric Profile}

Almost all patients (99.1\%) had at least 1 BPSD, and 71\% had 4 or more symptoms of BPSD. The overall NPI 12-item score was $27.42 \pm 18.24$ (range $0-72$ ). Of the individual BPSD domains, apathy/indifference was the most frequent (72.9\%), followed by agitation/ aggression (68.2\%), irritability/lability (59.8\%), sleep and nighttime behaviour disorders (58.9\%), depression/dysphoria (56.1\%), appetite and eating disorders (52.3\%), and anxiety (51.4\%). Aberrant motor behaviour (31.8\%), delusions (29\%), and hallucinations (23.4\%) were less frequent, while disinhibition (13.1\%) and elation/euphoria (9.3\%) were rare.

Although the number of BPSD present (considering the occurrence of $0 / 1 / 2 / 3 / \geq 4$ symptoms) was similar in the different types of dementia $(p=0.415)$, the magnitude of BPSD when measured by the overall NPI 12-item score was significantly higher in FTD followed by $\mathrm{DLB}$, lower in mixed dementia and $\mathrm{AD}$, and least in $\operatorname{VaD}(p=0.026)$. Hallucinations were more common in DLB (present in all cases) ( $p=0.008)$, and aberrant motor behaviour was more frequent in FTD (present in 68.42\% of cases) ( $p=0.003$ ) (Table 2). Other BPSD domains showed variations according to the types of dementia which did not reach statistical significance; e.g., FTD had more disinhibition while VaD had relatively less agitation and mixed dementia had less appetite and eating disorders. Among the various types of dementia, some BPSD had a frequency of $\geq 50 \%$ : AD patients had predominantly apathy, agitation, irritability, depression, sleep disorders and anxiety, FTD patients had agitation, apathy, appetite and eating disorders, aberrant motor behaviour, sleep disorders, and irritability, VaD patients had depression, anxiety, apathy, and irritability, mixed dementia patients had agitation, irritability, apathy, depression, and sleep disorders, and DLB patients had hallucinations, agitation, apathy, sleep disorders, delusions, depression, anxiety, irritability, and appetite and eating disorders (Fig. 1).

The overall number $(p<0.001)$ and magnitude $(p<0.001)$ of BPSD increased with the severity of dementia, and the NPI 12-item score showed strongly positive correlation with increasing CDR-SOB scores $\left(r_{\mathrm{S}}=0.626, p<0.001\right)$. All individual BPSD domains, except for anxiety and elation, showed a significant positive correlation with increasing severity of dementia (especially agitation, apathy, sleep disorders, appetite and eating disorders, and aberrant motor behaviour). Delusion, hallucinations, and depression were more prominent in moderate severity of dementia. Overall, BPSD along with all the individual domains, except for hallucinations, anxiety, elation, and aberrant motor behaviour, showed significant positive correlation with the duration of dementia. Similarly, BPSD showed a significant positive correlation with IADL CDI, except for anxiety and elation. Overall, BPSD $\left(r_{\mathrm{S}}=-0.239, p=\right.$ $0.013)$ and individually aberrant motor behaviour $\left(r_{\mathrm{S}}=-0.279, p=0.004\right)$ and apathy $\left(r_{\mathrm{S}}=\right.$ $-0.250, p=0.009$ ) showed significant negative correlation with age. Agitation was more common in males $(p=0.012)$. Apathy $(p=0.009)$ and appetite and eating disorders $(p=$ 0.025 ) were more prevalent in the rural community (Table 3 ). There was no difference in BPSD among the various levels of education. 
Dementia

and Geriatric
Dement Geriatr Cogn Disord Extra 2017;7:354-365

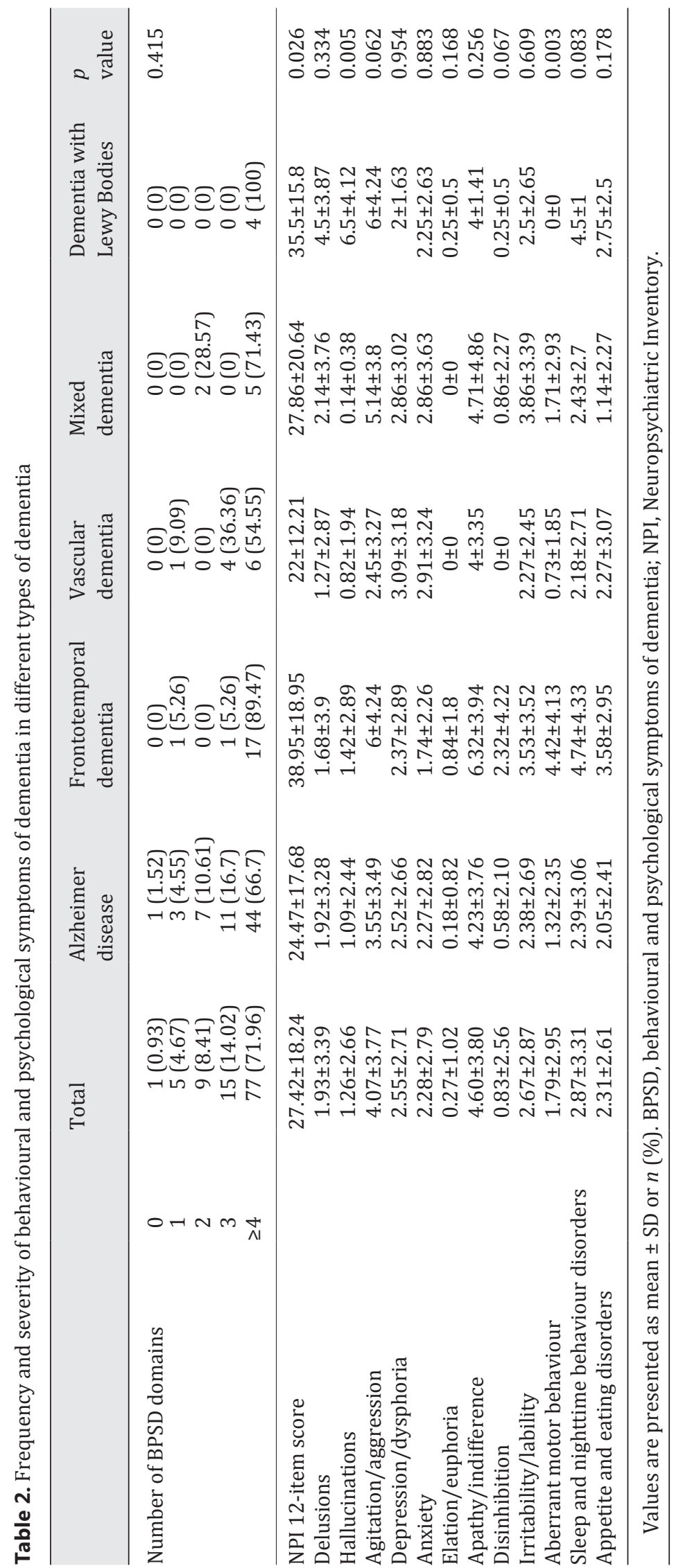

Mukherjee et al.: Behavioural and Psychological Symptoms of Dementia: Correlates and Impact on Caregiver Distress

C 2017 The Author(s). Published by S. Karger AG, Basel www.karger.com/dee

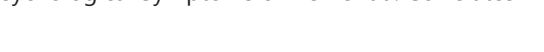




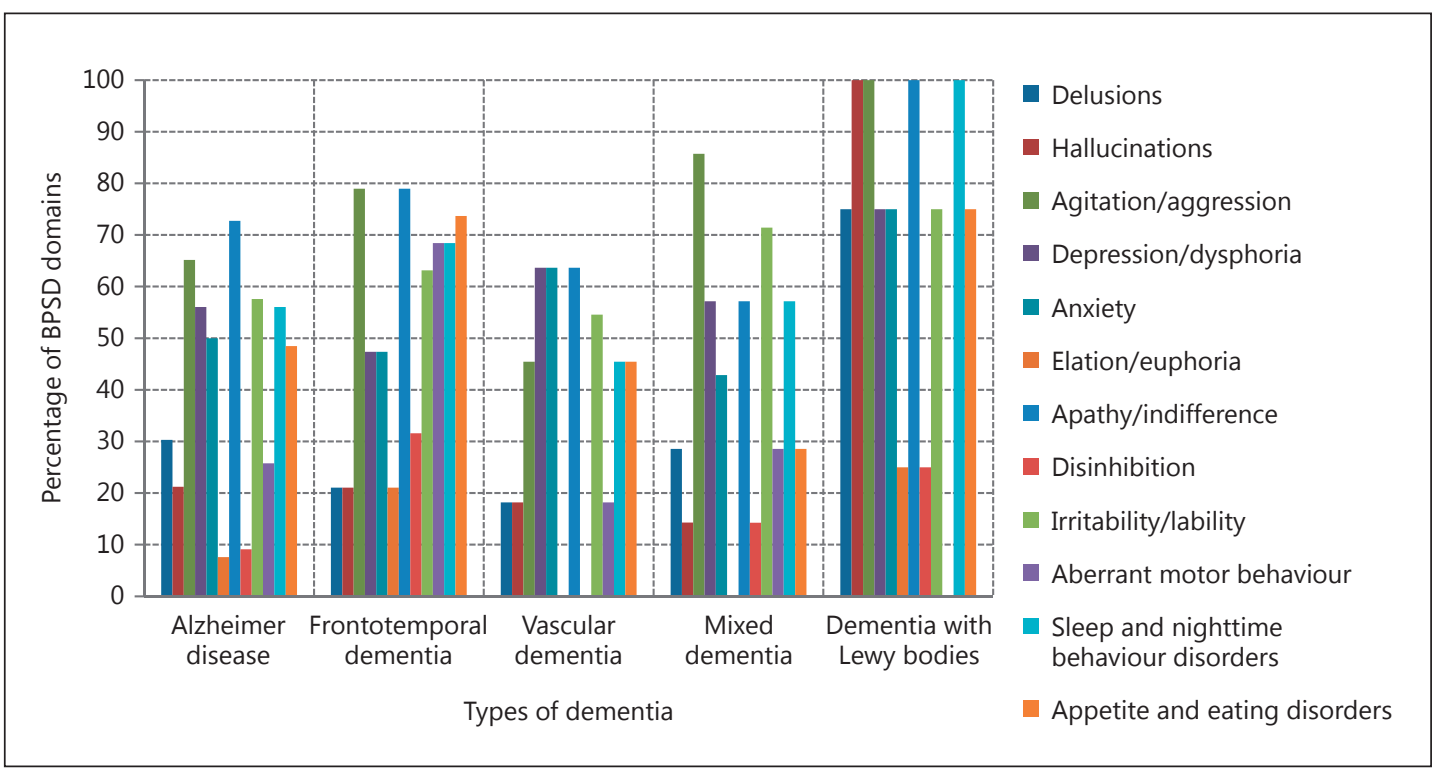

Fig. 1. Frequency of behavioural and psychological symptoms of dementia (BPSD) in different types of dementia.

\section{Caregiver Distress Associated with BPSD}

The overall NPI-D 12-item score was $12.73 \pm 7.82$. Caregiver distress increased with increasing number $(p<0.001)$ and magnitude $(p<0.001)$ of BPSD. On multiple linear regression analysis, all BPSD domains, except for elation, were significant predictors of caregiver distress (NPI-D) (Table 4).

\section{Discussion}

In this study, $\mathrm{AD}$ was the most common type of dementia followed by FTD, VaD, and mixed dementia, DLB being the rarest. Being a clinic-based study, this does not reflect the pattern in the community. Nevertheless, in contrast to data from western countries [26], a comparatively lower proportion of DLB and a higher proportion of VaD and FTD has also been reported from India [27]. The mean age at presentation was 66.6 years. Similarly, younger age at presentation of dementia has also been reported by other Indian studies [27]. AD had a greater proportion of female patients compared to other dementias; however, the overall majority of patients in this study were males. In this study, a majority $(77.6 \%)$ had mild to moderate dementia, while $15.9 \%$ had severe dementia and $6.5 \%$ had very mild dementia. The different types of dementia showed no significant difference regarding severity of dementia or impairment of IADL.

Almost every patient in our study (99.1\%) had at least 1 BPSD, and the majority had multiple such symptoms. This corroborates with previous studies $[1,3,10]$. Thus, BPSD are almost universally present in dementia patients if enquired properly. The most frequent BPSD in our study were apathy and agitation. Apathy and agitation have been found to be the most common neuropsychiatric symptoms in quite a few studies. Other common BPSD have been depression, anxiety, irritability, and sleep disorders [28-30], whereas euphoria/elation have been the least common symptoms [31]. It has further been described that nearly $50 \%$ 
Dementia

and Geriatric
Dement Geriatr Cogn Disord Extra 2017;7:354-365

(c) 2017 The Author(s). Published by S. Karger AG, Basel www.karger.com/dee

Mukherjee et al.: Behavioural and Psychological Symptoms of Dementia: Correlates and Impact on Caregiver Distress

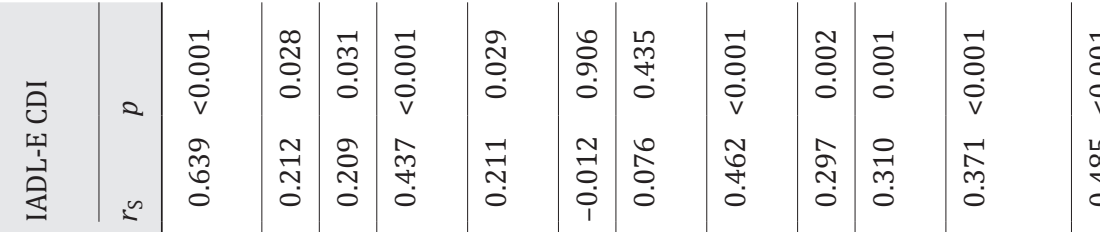

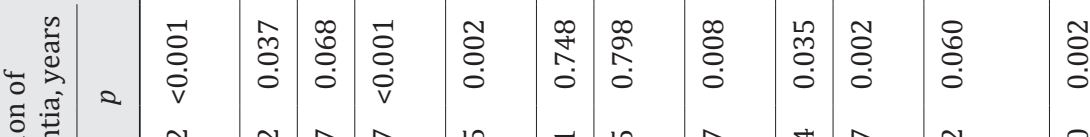

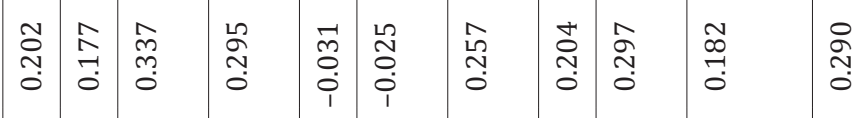

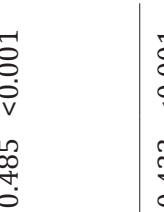

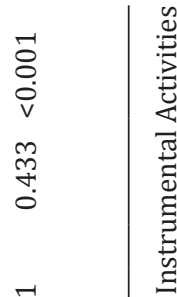

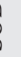

2

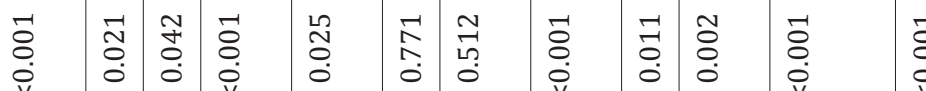

岂 $\approx$

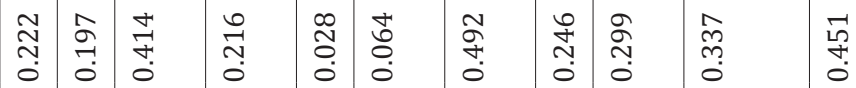

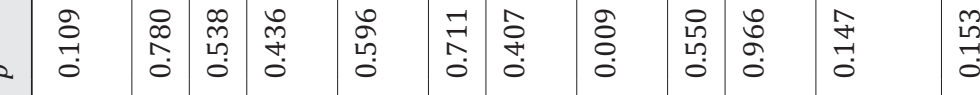

$\overbrace{0}^{\infty}$

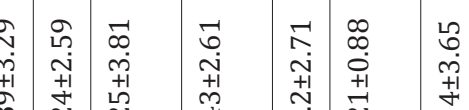

:

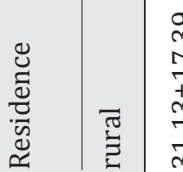

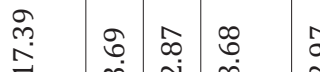

กิ

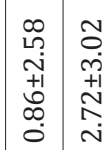

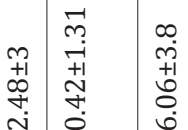

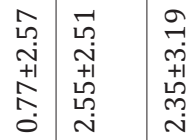

$m$
$\infty$
$\infty$
+1
$\dot{m}$
$\dot{m}$

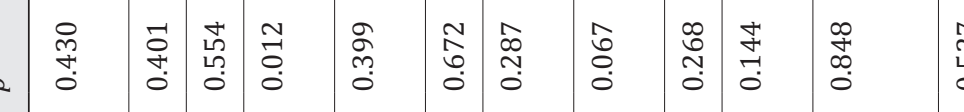

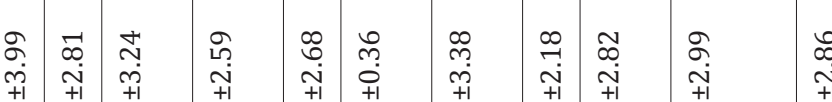

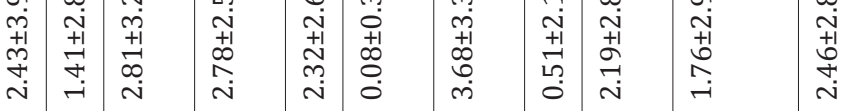

छัฒ

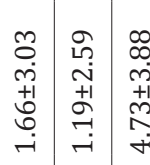

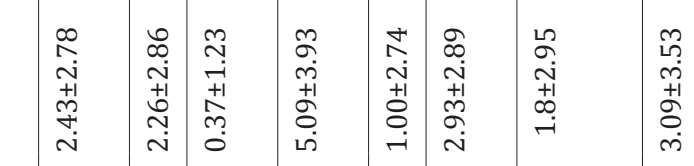

m

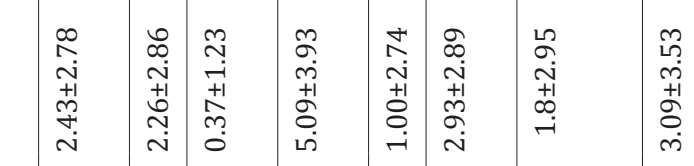

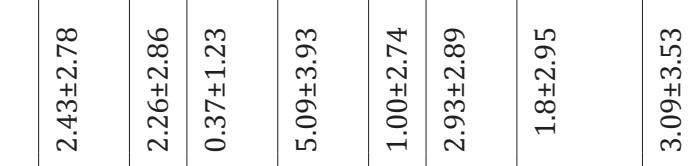

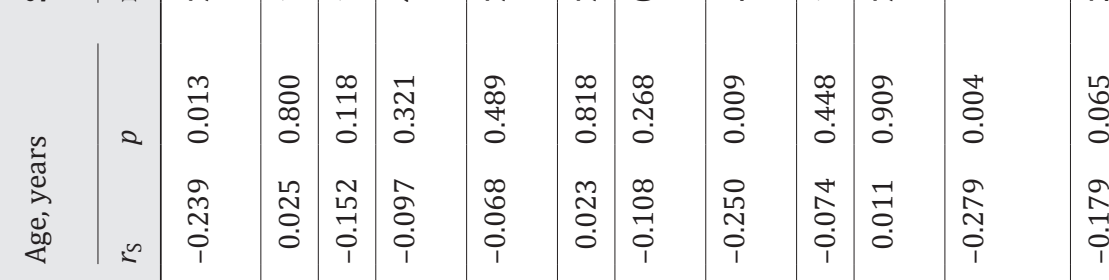


Table 4. Multiple linear regression analysis of behavioural and psychological symptoms of dementia as predictors of caregiver distress (NPI-D 12-item score)

\begin{tabular}{|c|c|c|c|c|c|}
\hline \multirow[t]{3}{*}{ Model } & \multicolumn{3}{|c|}{ Coefficients ${ }^{\mathrm{a}}$} & \multirow[t]{3}{*}{$t$} & \multirow{3}{*}{$\begin{array}{l}\text { Signif- } \\
\text { icance }\end{array}$} \\
\hline & \multicolumn{2}{|c|}{ unstandardised } & \multirow{2}{*}{$\frac{\text { standardised }}{\beta}$} & & \\
\hline & $\mathrm{B}$ & SE & & & \\
\hline (Constant) & 1.071 & 0.386 & & 2.774 & 0.007 \\
\hline Delusions & 0.418 & 0.065 & 0.181 & 6.409 & 0.000 \\
\hline Hallucinations & 0.429 & 0.082 & 0.146 & 5.256 & 0.000 \\
\hline Agitation/aggression & 0.398 & 0.066 & 0.192 & 6.062 & 0.000 \\
\hline Depression/dysphoria & 0.428 & 0.078 & 0.148 & 5.465 & 0.000 \\
\hline Anxiety & 0.451 & 0.072 & 0.161 & 6.251 & 0.000 \\
\hline Elation/euphoria & 0.245 & 0.215 & 0.032 & 1.141 & 0.257 \\
\hline Apathy/indifference & 0.445 & 0.056 & 0.216 & 7.919 & 0.000 \\
\hline Disinhibition & 0.365 & 0.111 & 0.119 & 3.296 & 0.001 \\
\hline Irritability/lability & 0.562 & 0.090 & 0.206 & 6.217 & 0.000 \\
\hline Aberrant motor behaviour & 0.494 & 0.074 & 0.186 & 6.633 & 0.000 \\
\hline Sleep and nighttime behaviour disorders & 0.322 & 0.081 & 0.137 & 3.980 & 0.000 \\
\hline Appetite and eating disorders & 0.369 & 0.086 & 0.123 & 4.308 & 0.000 \\
\hline
\end{tabular}

NPI-D, Neuropsychiatric Inventory distress scale; SE, standard error. ${ }^{a}$ Dependent variable: NPI-D 12 -item score.

of dementia patients have at least 4 neuropsychiatric symptoms simultaneously [8]. Our study showed similar results, with $71 \%$ patients having $\geq 4$ neuropsychiatric symptoms.

The overall magnitude of BPSD was higher in FTD followed by DLB, lower in mixed dementia and $\mathrm{AD}$, and least in $\mathrm{VaD}$. This is understandable because in FTD, the fronto-subcortical networks responsible for various behavioural symptoms are involved early, and in DLB, the psychiatric manifestations form an integral part of the diagnosis. Other studies have similarly shown a higher degree of BPSD in DLB and FTD [1,32]. Our study showed less BPSD in VaD. Although there are reports contrary to this [1], other Indian studies have showed less BPSD in VaD compared to AD [9].

In our study, hallucinations were more common in DLB and aberrant motor behaviour was more frequent in FTD. Although statistical significance was not achieved, disinhibition was more common in FTD, while agitation and eating disorders were less common in VaD and mixed dementia, respectively. A study on dementia type-specific BPSD showed predominance of hallucinations in DLB, activity disturbances in FTD, anxiety and phobias in AD, and affective disturbance in $\mathrm{VaD}$ [1]. An Indian study noted that aberrant motor behaviour, disinhibition, and appetite/eating disturbances could differentiate FTD from AD and VaD, but found no difference between $\mathrm{AD}$ and $\mathrm{VaD}$ [12]. We also observed some pattern in BPSD in dementia subtypes. While AD patients predominantly showed apathy, agitation, irritability, depression, sleep disorders, and anxiety, FTD patients showed agitation, apathy, appetite and eating disorders, aberrant motor behaviour, sleep disorders, irritability, disinhibition, and elation; VaD patients had depression, anxiety, apathy, and irritability; mixed dementia patients showed agitation, irritability, apathy, depression, and sleep disorders; DLB patients had hallucinations, agitation, apathy, sleep disorders, delusions, depression, anxiety, irritability, and appetite and eating disorders. Visual hallucinations are one of the core diagnostic features of DLB, hence their abundance. Preferential disruption of the orbitofrontal-subcortical circuit in FTD might explain the prominence of symptoms such as disinhibition. As for most of the other symptoms, the lack of any significant difference among the dementia 
Mukherjee et al.: Behavioural and Psychological Symptoms of Dementia: Correlates and Impact on Caregiver Distress

subtypes indicates that the neuroanatomical substrates for these BPSD are involved in all types of dementia. This may be explained by the involvement of different components of the same cognitive-behavioural networks depending on the predominant cortical/subcortical location of the pathology among the different types of dementia. Thus, in our study, although the BPSD symptoms formed clusters according to the different types of dementia, other than hallucinations in DLB, aberrant motor behaviour and to some extent disinhibition in FTD, other symptoms of BPSD were more or less overlapping and probably not very disease specific.

All BPSD domains, except for anxiety and elation/euphoria, were more prominent with increasing severity of dementia. The individual domains were also more prominent with increasing duration of dementia, except for hallucinations, anxiety, elation, and aberrant motor behaviour. Higher numbers of BPSD in advanced stages of dementia have been also demonstrated in other studies $[1,28,33]$. Moreover, similar to our study, these studies have shown that anxiety and euphoria do not follow this pattern $[28,33]$. Interestingly, delusion, hallucinations, and depression were most prominent in moderate severity of dementia in our study. One possible explanation of this may be that the appearance of other more prominent BPSD in advanced dementia might falsely suppress reporting of these symptoms, e.g., severe apathy in advanced dementia may lead to underexpression of psychotic phenomenon. It could also be because of improvement of some of these symptoms either spontaneously or with medication in advanced stages of dementia.

BPSD, except for anxiety and elation, increased in severity with deterioration of activities of daily living. Other studies have also noted an association between impairment of activities of daily living and presence of neuropsychiatric symptoms [4]. In our study, overall BPSD and the domains of aberrant motor behaviour and apathy were more prominent in younger patients. An Indian study noted similarly that BPSD correlated inversely with age [10]. A meta-analysis on AD concluded that age could affect the prevalence of many BPSD, such as aggression, disinhibition, anxiety, irritability, depression, euphoria, and delusion [5]. In our study, the greater proportion of FTD in the younger patients may have led to the higher BPSD, especially aberrant motor behaviour. However, the age-related difference for some BPSD may be due to underreporting in the elderly, such as apathy. This may have been considered normal by some caregivers, as the general concept of our society is that older people are probably quieter. Males in our study showed more agitation/aggression. Other studies have shown a higher prevalence of physical aggression, aberrant motor behaviour, and apathy in men, while women displayed more anxiety, depression, and delusions; however, some studies have not shown any correlation with age or gender [3]. The excess of agitation/aggression in males may be due to the inherent gender-based psychological differences or to the genderdependent differential neuropathological involvement in dementia [34]. However, as discussed by Brodaty et al. [28], it may be a reflection of socialised gender roles tolerating greater disruption from males.

We found no difference in BPSD with education. However, our study showed that apathy and eating disorders were more prevalent in the rural subjects. Apathy in younger subject gets noticed easily as compared to that in the old, in whom it is considered normal by society. Since our rural cohort had a higher number of younger subjects, the greater presence of apathy could be ascribed to this. However, the increased prevalence of eating disorders in the rural subjects is not explainable. More elaborate community-based studies are needed to draw a definite conclusion.

Our study demonstrated that caregiver distress increased with increasing number and magnitude of BPSD. Other studies have also shown a significant association between BPSD and high levels of caregiver distress [10,30], especially with delusion and agitation/aggression $[31,35]$. We found, on multiple linear regression analysis, that elation/euphoria was the only 
individual domain of BPSD not significantly predicting caregiver distress. Elation/euphoria has been found to be less distressing in other studies also [35]. It may be that the caregivers do not feel distressed as the mood in euphoria is seemingly cheerful and the patient appears happy despite the disease. Overall, however, BPSD are a major cause of caregiver distress. Caring for a dementia patient itself being a demanding task, it is understandable that coping with the additional burden of neuropsychiatric symptoms leads to significant distress for caregivers.

\section{Limitations of the Study}

This is a clinic-based study conducted in a tertiary care hospital, which may have led to referral bias. This, along with the small sample size, limits the generalisability of the results. This being a cross-sectional study, causal relationships cannot be definitively established. We diagnosed the types of dementia based on clinical criteria supported by radiological parameters, but did not use pathological (autopsy) confirmation. However, as the criteria used were well established, we feel that they should be sufficient for all practical purposes of this clinical study.

\section{Conclusions}

In this study, BPSD were almost universally present in dementia patients, and the majority had multiple symptoms. The most frequent BPSD in our study were apathy and agitation, followed by irritability, sleep and nighttime behaviour disorders, depression, appetite and eating disorders, and anxiety, whereas disinhibition and elation/euphoria were least frequent. BPSD were most numerous in FTD followed by DLB and VaD. We observed some patterns of clustering of BPSD according to various dementia subtypes. However, other than hallucinations in DLB and aberrant motor behaviour (and to some extent disinhibition) in FTD, other symptoms of BPSD were overlapping and probably not disease specific. All BPSD domains, except for anxiety and elation/euphoria, were more prominent with increasing severity of dementia. Overall, BPSD were more prominent in younger patients. Males in our study showed more agitation/aggression compared to females. Although our study showed apathy and eating disorders to be more prevalent in patients from the rural community, this observation requires confirmation from community-based studies. This study demonstrated that caregiver distress increased with increasing number of BPSD, and elation/euphoria was the only individual domain of BPSD not significantly predicting caregiver distress.

\section{Disclosure Statement}

The corresponding author certifies that there is no conflict of interest and that all authors have read the paper and have agreed to be listed as authors.

\section{References}

1 Chiu MJ, Chen TF, Yip PK, Hua MS, Tang LY: Behavioral and psychologic symptoms in different types of dementia. J Formos Med Assoc 2006;105:556-562.

2 Shimabukuro J, Awata S, Matsuoka H: Behavioral and psychological symptoms of dementia characteristic of mild Alzheimer patients. Psychiatry Clin Neurosci 2005;59:274-279.

3 Ford AH: Neuropsychiatric aspects of dementia. Maturitas 2014;79:209-215.

$\checkmark 4$ McKeith I, Cummings J: Behavioural changes and psychological symptoms in dementia disorders. Lancet Neurol 2005;4:735-742. 
5 Zhao QF, Tan L, Wang HF, et al: The prevalence of neuropsychiatric symptoms in Alzheimer's disease: systematic review and meta-analysis. J Affect Disord 2016;190:264-271.

6 Brodaty H, Connors MH, Xu J, Woodward M, Ames D; PRIME Study Group: Predictors of institutionalization in dementia: a three year longitudinal study. J Alzheimers Dis 2014;40:221-226.

7 Tan LL, Wong HB, Allen H: The impact of neuropsychiatric symptoms of dementia on distress in family and professional caregivers in Singapore. Int Psychogeriatr 2005;17:253-263.

-8 Cerejeira J, Lagarto L, Mukaetova-Ladinska EB: Behavioral and psychological symptoms of dementia. Front Neurol 2012;3:73.

-9 Pinto C, Seethalakshmi R: Behavioral and psychological symptoms of dementia in an Indian population: comparison between Alzheimer's disease and vascular dementia. Int Psychogeriatr 2006;18:87-93.

$>10$ Shaji KS, George RK, Prince MJ, Jacob KS: Behavioral symptoms and caregiver burden in dementia. Indian J Psychiatry 2009;51:45-49.

11 Shaji S, Bose S, Kuriakose S: Behavioral and psychological symptoms of dementia: a study of symptomatology. Indian J Psychiatry 2009;51:38-41.

12 Srikanth S, Nagaraja AV, Ratnavalli E: Neuropsychiatric symptoms in dementia-frequency, relationship to dementia severity and comparison in Alzheimer's disease, vascular dementia and frontotemporal dementia. J Neurol Sci 2005;236:43-48.

13 American Psychiatric Association: Diagnostic and Statistical Manual of Mental Disorders, ed 4. Washington, DC, American Psychiatric Association, 1994.

14 McKhann G, Drachman D, Folstein M, et al: Clinical diagnosis of Alzheimer's disease: report of the NINCDSADRDA Work Group under the auspices of Department of Health and Human Services Task Force on Alzheimer's Disease. Neurology 1984;34:939-944.

15 Rascovsky K, Hodges JR, Knopman D, et al: Sensitivity of revised diagnostic criteria for the behavioural variant of frontotemporal dementia. Brain 2011;134:2456-2477.

16 Gorno-Tempini ML, Hillis AE, Weintraub S, et al: Classification of primary progressive aphasia and its variants. Neurology 2011;76:1006-1014.

17 McKeith IG, Dickson DW, Lowe J, et al: Diagnosis and management of dementia with Lewy bodies: third report of the DLB Consortium. Neurology 2005;65:1863-1872.

18 Román GC, Tatemichi TK, Erkinjuntti T, et al: Vascular dementia: diagnostic criteria for research studies. Report of the NINDS-AIREN International Workshop. Neurology 1993;43:250-260.

19 Hughes CP, Berg L, Danziger WL, et al: A new clinical scale for the staging of dementia. Br J Psychiatry 1982; 140:566-572.

20 Morris JC: The Clinical Dementia Rating (CDR): current vision and scoring rules. Neurology 1993;43:2412-2414.

-21 Mathuranath PS, George A, Cherian PJ, et al: Instrumental activities of daily living scale for dementia screening in elderly people. Int Psychogeriatr 2005;17:461-474.

22 Cummings JL, Mega M, Gray K, Rosenberg-Thompson S, Carusi DA, Gornbein J: The Neuropsychiatric Inventory: comprehensive assessment of psychopathology in dementia. Neurology 1994;44:2308-2314.

23 Cummings JL: The Neuropsychiatric Inventory: assessing psychopathology in dementia patients. Neurology 1997;48(suppl 6):S10-S16.

-24 Kaufer DI, Cummings JL, Christine D, et al: Assessing the impact of neuropsychiatric symptoms in Alzheimer's disease: the Neuropsychiatric Inventory Caregiver Distress Scale. J Am Geriatr Soc 1998;46:210-215.

-25 Bandyopadhyay TK, Biswas A, Roy A, et al: Neuropsychiatric profiles in patients with Alzheimer's disease and vascular dementia. Ann Indian Acad Neurol 2014;17:325-330.

-26 Barker WW, Luis CA, Kashuba A, et al: Relative frequencies of Alzheimer disease, Lewy body, vascular and frontotemporal dementia, and hippocampal sclerosis in the State of Florida Brain Bank. Alzheimer Dis Assoc Disord 2002;16:203-212.

27 Alladi S, Mekala S, Chadalawada SK, Jala S, Mridula R, Kaul S: Subtypes of dementia: a study from a memory clinic in India. Dement Geriatr Cogn Disord 2011;32:32-38.

28 Brodaty H, Connors MH, Xu J, Woodward M, Ames D; PRIME Study Group: The course of neuropsychiatric symptoms in dementia: a 3-year longitudinal study. J Am Med Dir Assoc 2015;16:380-387.

29 Lyketsos CG, Lopez O, Jones B, Fitzpatrick AL, Breitner J, DeKosky S: Prevalence of neuropsychiatric symptoms in dementia and mild cognitive impairment: results from the Cardiovascular Health Study. JAMA 2002;288: 1475-1483.

-30 Rosdinom R, Zarina MZ, Zanariah MS, Marhani M, Suzaily W: Behavioural and psychological symptoms of dementia, cognitive impairment and caregiver burden in patients with dementia. Prev Med 2013;57(suppl):S67-S69.

-31 Huang SS, Lee MC, Liao YC, Wang WF, Lai TJ: Caregiver burden associated with behavioral and psychological symptoms of dementia (BPSD) in Taiwanese elderly. Arch Gerontol Geriatr 2012;55:55-59.

-32 Bjoerke-Bertheussen J, Ehrt U, Rongve A, Ballard C, Aarsland D: Neuropsychiatric symptoms in mild dementia with Lewy bodies and Alzheimer's disease. Dement Geriatr Cogn Disord 2012;34:1-6.

33 Selbaek G, Engedal K, Benth JŠ, Bergh S: The course of neuropsychiatric symptoms in nursing-home patients with dementia over a 53-month follow-up period. Int Psychogeriatr 2014;26:81-91.

-34 Mazure CM, Swendsen J: Sex differences in Alzheimer's disease and other dementias. Lancet Neurol 2016;15: 451-452.

35 Song JA, Oh Y: The association between the burden on formal caregivers and behavioral and psychological symptoms of dementia (BPSD) in Korean elderly in nursing homes. Arch Psychiatr Nurs 2015;29:346-354. 